\title{
Lesions of the Amygdala Central Nucleus Alter Performance on a Selective Attention Task
}

\author{
Peter C. Holland, ${ }^{1}$ Jung-Soo Han, ${ }^{2}$ and Michela Gallagher ${ }^{2}$ \\ ${ }^{1}$ Department of Psychology, Duke University, Durham, North Carolina, 27708, and 2Department of Psychology, \\ Johns Hopkins University, Baltimore, Maryland 21218
}

\begin{abstract}
Previous studies showed a role for the amygdala central nucleus (CN) in attentional processing during the acquisition of Pavlovian associations. Both the acquisition of conditioned orienting responses and the surprise-induced enhancement in the ability of conditioned stimuli to enter into new associations depend on the integrity of $\mathrm{CN}$. In this experiment, the role of $\mathrm{CN}$ in the performance of a well-learned selective attention task was examined. Rats with ibotenic acid lesions of $\mathrm{CN}$ and control rats first learned a discrete-trial, multiple-choice reaction time task. On each trial, after a constant-duration ready signal, the rats were required to poke their noses into one of three ports, guided by the brief illumination of one of those ports. Rats with $\mathrm{CN}$ lesions were slower to acquire the task than control rats but showed equiva-
\end{abstract}

lent asymptotic sustained performance. Subsequent attentional challenges, which included reducing the duration of the port illumination and varying the duration of the ready signal, had greater impact on the performance of lesioned than control rats. These data resemble those reported from similar tasks after damage to the basal forebrain (BF) system. Together with earlier findings, these data support a role for $\mathrm{CN}$ in modulating visuospatial attention in action as well as in the acquisition of associations, perhaps by way of its projections to BF cholinergic systems.

Key words: amygdala; central nucleus; selective attention; basal forebrain cholinergic system; reaction time; rats
Recent data implicate the amygdala central nucleus $(\mathrm{CN})$ in the modulation of attention during the acquisition of Pavlovian conditioning (for review, see Holland and Gallagher, 1999). First, the acquisition of conditioned orienting behavior (OR) to conditioned stimuli (CSs) paired with food depends on the integrity of amygdalonigrostriatal circuitry. Rats with bilateral lesions of CN (Gallagher et al., 1990) or with asymmetric lesions that disconnect CN from dorsolateral striatum (Han et al., 1997) fail to acquire these conditioned ORs. Second, neural circuitry that includes CN connections with basal forebrain (BF) structures is important for surprise-induced enhancements in the ability of CSs to enter into new associations, an attentional property known as "associability" (Pearce and Hall, 1980). These enhancements are abolished in rats by bilateral lesions of CN (Holland and Gallagher, 1993a,b), bilateral lesions of the large cholinergic neurons of the substantia innominata/nucleus basalis magnocellularis (SI/nBM; Chiba et al., 1995), and asymmetric lesions that disconnect CN from those SI/nBM neurons (Han et al., 1999).

Other research demonstrates a role for the BF cholinergic system in the regulation of attentional processes engaged in sustained or selective attention tasks (Muir et al., 1992; Pang et al., 1993; Voytko et al., 1994; McGaughy et al., 1996; Chiba et al., 1999). In these tasks, animals perform previously learned responses under conditions thought to place demands on attentional processing of the stimuli that guide responding. For example, in the multiplechoice reaction time (MCRT) task (Carli et al., 1983), rats must nose poke into one of several ports when it is briefly illuminated. Performance in this task requires selection of one of many elements of the stimulus (port) array. Manipulations designed to increase attentional demands typically depress performance. Notably, rats with lesions of the BF cholinergic system show impaired performance on these tasks, especially under conditions of high

\footnotetext{
Received March 3, 2000; revised June 12, 2000; accepted June 13, 2000.

This research was supported by Grant MH-53667 and Research Scientist Award K08 MH01149 (M.G.) from the National Institute of Mental Health. We thank Helena Winfield for technical assistance.

Correspondence should be addressed to Peter Holland, Department of Psychology: Experimental, Duke University, Box 90086, Durham, NC 27708. E-mail: pch@ duke.edu.

Copyright (C) 2000 Society for Neuroscience $0270-6474 / 00 / 206701-06 \$ 15.00 / 0$
}

attentional demand (Robbins et al., 1989; Muir et al., 1992, 1994, 1996; Waite et al., 1999).

The question addressed in this study is whether performance on the MCRT task is affected by $\mathrm{CN}$ lesions. Although it seems plausible that attentional processes determining performance in this task and those affecting acquisition of new learning (known to be affected by $\mathrm{CN}$ lesions) might engage similar brain circuitry, there is reason to think otherwise. For example, considerable data (Hall and Pearce, 1979; Holland and Gallagher, 1993a) support the claim of a popular associative learning theory (Pearce and Hall, 1980 ) that changes in the associability of a cue are independent of its ability to control behavioral performance. Although BF cholinergic neurons may be involved in both surprise-induced associability enhancements and response selection in attentional tasks such as the MCRT, CN might be critical only for the former.

Rats with ibotenic acid lesions of $\mathrm{CN}$ and control rats were first trained on a simplified version of the MCRT task. After performance on the task stabilized, rats were tested under three conditions of attentional challenge: the reduction of signal duration, the occurrence of auditory distracters, and the introduction of variability in the time of signal presentation.

\section{MATERIALS AND METHODS}

Subjects, surgery, and histological analysis. The subjects were 16 male Long-Evans rats (Charles River, Raleigh, NC) that weighed 300-325 gm at the beginning of the experiment. After the surgical procedure but before the behavioral manipulations of this study, they participated in a conditioning experiment in behavioral test chambers that differed in size, construction, and location from those used in this study. The rats lived in individual cages with ad libitum access to water and were maintained at $85 \%$ of their ad libitum weights by limiting their access to food. The colony room was illuminated from 6 A.M. to 8 P.M.

Bilateral CN lesions were made in eight rats by injecting $0.2 \mu$ l of ibotenic acid (Sigma, St. Louis, MO), dissolved in PBS $(10 \mu \mathrm{g} / \mu \mathrm{l})$ at a single site in each hemisphere (anteroposterior, -2.3 ; mediolateral, \pm 4.2 ; and dorsoventral, -7.7). Eight control rats received injections of the same volume of PBS vehicle alone. Injections were made with a Hamilton microsyringe equipped with a 27 gauge needle.

Histological procedures. After completing the behavioral experiments, the rats were killed by overdose with pentobarbital $(100 \mathrm{mg} / \mathrm{kg})$ and perfused with $4 \%$ paraformaldehyde. Brains were removed, post-fixed in perfusate for $2 \mathrm{hr}$, cryoprotected ( $20 \%$ sucrose), and sectioned (coronal plane) on a freezing microtome. Adjacent tissue sections through BF $(40 \mu \mathrm{m})$ were taken from each brain. In each set of four serial sections, 
every first, second, and third section was processed for choline acetyltransferase (ChAT), parvalbumin immunoreactivity, and acetycholinesterase (AChE) histochemistry, respectively, and every fourth section was mounted on slides for Nissl stain to verify the neurotoxic lesion of $\mathrm{CN}$.

For ChAT and parvalbumin immunoreactivity, endogenous peroxidase within the tissue was blocked by washing the sections for $30 \mathrm{~min}$ in a solution of $3 \% \mathrm{H}_{2} \mathrm{O}_{2}$ and $10 \% \mathrm{MeOH}$ in $0.1 \mathrm{M}$ PBS, followed by two $5 \mathrm{~min}$ washes in PBS with $0.3 \%$ Triton-X (PBST). To block background (nonspecific) staining, the sections were incubated for $1 \mathrm{hr}$ in a solution of PBST with a $10 \%$ concentration of the appropriate serum. To visualize ChATand parvalbumin-positive cells in BF, sections were then incubated with the appropriate antibody (ChAT, 1:200 dilution; Chemicon, Temecula, CA; parvalbumin, 1:1000 dilution; Sigma) overnight at $4^{\circ} \mathrm{C}$. The next day, sections were rinsed twice in PBST, incubated for $1 \mathrm{hr}$ at room temperature in the appropriate biotinylated secondary antibody (1:200 dilution), rinsed twice again in PBST, and then incubated for $1 \mathrm{hr}$ at room temperature in ExtrAvidin peroxidase conjugate (1:1000 dilution; Sigma). After two PBS rinses for $5 \mathrm{~min}$ each, tissue was reacted using a Vector Laboratories (Burlingame, CA) SG substrate kit for peroxidase. Tissue sections were then mounted onto Superfrost slides, dehydrated through washes in ascending concentrations of alcohol, and coverslipped with Permount. The AChE staining procedure was adapted from that of Karnovsky and Roots (1964).

Integrity of the basal forebrain cholinergic neurons was examined by comparison of ChAT-immunostained sections from control rats with anatomically matched sections from rats in the $\mathrm{CN}$ lesion group. The extent of the neurotoxic lesion of $\mathrm{CN}$ was determined in Nissl-stained sections. For each section through the rostrocaudal extent of the nucleus, the percentage of $\mathrm{CN}$ area in which neurons were absent was estimated for each side separately. Note that this criterion provides a conservative estimate of $\mathrm{CN}$ damage, because surrounding the region in which no intact neurons are visible, neurons are typically more sparse as the toxin diff uses from the injection site. For the lesion site in each hemisphere, the average of the extent of lesion was calculated across sections. A lesion was determined to be acceptable if lesion size encompassed at least $30 \%$ of the nucleus; rats were included in the data analysis only if this minimum size was achieved bilaterally.

Behavioral apparatus. The test apparatus comprised four $26.5 \times 26.5 \times$ $26.5 \mathrm{~cm}$, nine-hole reaction time chambers (Paul Fray, Cambridge, UK) like those used by Muir et al. (1994). The curved stainless steel front wall of each chamber contained nine $2.5 \times 2.5 \mathrm{~cm}$ ports, all but three of which were blocked with an opaque insert. The three ports used were those in the center of the wall and $2.5 \mathrm{~cm}$ to the right and left of center, $2 \mathrm{~cm}$ above the mesh floor. Each port included an infrared phototransistor to detect nose pokes and could be illuminated by a $3 \mathrm{~W}$ bulb at the back of the port. A recessed food cup, covered by a hinged acrylic plastic door, was located in the center of the rear wall of the chamber, $2 \mathrm{~cm}$ from the floor. A $3 \mathrm{~W}$ house light and a speaker were mounted in the center of the top wall.

Behavioral procedures. The rats were first familiarized with the apparatus in four sessions. In the first two 15 min sessions, the response ports were covered, food pellets ( $45 \mathrm{mg}$; Noyes, Lancaster, $\mathrm{NH}$ ) were present in the food cup, and the acrylic door to the food cup was propped open. In the next two $30 \mathrm{~min}$ sessions, the response ports were open, and food pellets were placed in each response port as well as in the food cup.

In the baseline task used here, the beginning of a trial was signaled at random intervals by the illumination of the house light. After a constan $5 \mathrm{sec}$ ready period, one of the three target ports was illuminated for $0.5 \mathrm{sec}$. Each port was equally likely to be illuminated on any trial. The first response to the correct port within $5 \mathrm{sec}$ of port illumination was reinforced with the delivery of a food pellet to the food cup (accompanied by a $0.3 \mathrm{sec}$ illumination of the food cup) and the darkening of both the port (if still illuminated) and the house light. If no correct response was made before the end of the $5 \mathrm{sec}$ response window, the house light was darkened, and the trial ended. Responses to the ports that were not illuminated on a trial were recorded as errors and had no scheduled consequences. Trials were presented in random order at predetermined intervals within the 30 min sessions; trial delivery was not affected by the rats' behavior. Each rat received two sessions daily, the first at $\sim 7$ A.M. and the second at $\sim 3$ P.M.

Rats were shaped to this procedure gradually, but all rats received the same treatment (the shaping was not individualized). The duration of port illumination was reduced between sessions, from $30 \mathrm{sec}$ in the first two training sessions to $0.5 \mathrm{sec}$ in the last two sessions, with the values 30,25 , $20,15,10,5,3,2,1$, and $0.5 \mathrm{sec}$. Each duration was used in two consecutive sessions. In the first two sessions there were 10 of each trial type; in sessions 3-6 there were 12 of each type; in sessions 7 and 8 there were 15 of each type; and in all subsequent training sessions there were 20 of each type. When the target duration was $\geq 5 \mathrm{sec}$, responses were effective throughout the illumination of the target stimuli.

Three additional acquisition sessions with $0.5 \mathrm{sec}$ target cues provided an initial behavioral baseline for assessing the effects of attentional challenges. In the first two attentional challenge sessions, the target duration was reduced to $0.25 \mathrm{sec}$; otherwise these sessions were identical to the training sessions. In the next two sessions, distracter stimuli were added during the $5 \mathrm{sec}$ ready period. The distracters were the $1 \mathrm{sec}$ presentation of an $80 \mathrm{~dB}$ white noise during the first, third, and fifth seconds of the $5 \mathrm{sec}$ ready period and during the $0.25 \mathrm{sec}$ target cue presentations. In the fifth test session the rats were returned to the original $0.5 \mathrm{sec}$ target condition, with a session that was identical to the final training session. This session served as a baseline to assess the effects of the last two challenge sessions, in which the ready period was made variable. These two sessions were identical to the baseline session, except that the ready periods for each trial type were equally likely to be 1,5 , or $9 \mathrm{sec}$ in duration. A final session in this series served as a return to the constant ready-time baseline and was identical to the fifth test session.

At the end of the challenge sessions, the rats were given ad libitum access to food in their home cages for 1 week. The rats were then placed in the experimental chambers for another test, identical to the baseline sessions of the previous phase. This test was intended to assess the effect of a manipulation that would depress the level of responding, without necessarily affecting selective attention. Previous data suggest that $\mathrm{CN}$ lesions do not influence the effects of satiation on several aspects of Pavlovian appetitively conditioned responding (Gallagher and Holland, 1992; Hatfield et al., 1996).

This MCRT procedure differed from most previous versions of the task. First, only three stimulus and response ports were used in this experiment, rather than the five or more used in previous studies. Second, in this experiment, trial delivery was completely under the experimenter's control, rather than the subject's. Following the suggestion of Bushnell (1998, p 247), trials were initiated by a scheduled ready signal. In the usual MCRT protocol, each subject's behavior influences the initiation, postponement, and termination of trials. These additional operant contingencies increase the complexity of the task and might generate substantial between-subject variability in the sequencing and spacing of trials. Furthermore, this variability could potentially be confounded with lesion treatment if lesions affected the subjects' ability to master these contingencies. Minimizing the subjects' control over event scheduling, as in this study, makes such confounds less likely. Third, simplification of the task made it possible for each rat to learn without individualized shaping. In most previous studies with the MCRT procedure, the training parameters were adjusted as necessary for each rat during acquisition (Robbins et al., 1989; Muir et al., 1992, 1994; Waite et al., 1999); as a result, the rats may have been exposed to very different initial learning contingencies. Thus, our simplified MCRT procedure may provide an assessment of visuospatial attention less confounded by other factors. On the other hand, it could also be argued that our simplifications may also have substantially reduced the attentional demands of the task. Nonetheless, the conditions used were sufficiently sensitive to detect the effects of $\mathrm{CN}$ lesions when attentional demands were increased.

Behavioral data analysis. Behavioral performance was assessed with several measures. The primary measure was the latency to the first correct response, defined as the time between the onset of the target stimulus and the breaking of the photobeam in that port. If no correct response occurred on a trial, a maximum latency value was assigned. When the target duration was $<5 \mathrm{sec}$, that maximum latency was $5 \mathrm{sec}$, the response window duration; when the target duration was $>5 \mathrm{sec}$, the maximum latency was defined as equal to the target duration. Thus, this measure might be viewed as a composite measure of performance, influenced somewhat by the accuracy of responding as well as the speed of responding. When the accuracy of responding was substantially $<100 \%$, this measure was supplemented by two additional latency measures: the latency to the first correct response, limited to trials on which correct responding occurred, and the latency to the first correct response on trials on which the first response to occur was the correct response.

Two measures of accuracy were computed: the percentage of trials on which at least one correct response occurred and the percentage of trials on which the first response was the correct response. In addition, the latency to the first error and the percentage of trials on which errors occurred were recorded. Finally, two aspects of anticipatory responding were recorded: the number of responses to all ports (combined) during the ready signal but before the illumination of a target stimulus and the latency between the onset of the ready signal and the first anticipatory response.

Separate multivariate ANOVAs for each of the response measures, which used lesion condition, session, target cue (left, center, or right), and port response (left, center, or right), showed that there were no biases to respond to any of the cues or to perform any of the port responses in either lesion condition. Consequently, each measure was collapsed across target cue and port response and analyzed with univariate ANOVAs.

\section{RESULTS}

\section{Histological results}

Nissl staining showed acceptable bilateral lesions in six of the eight lesioned rats; behavioral data from the remaining two lesioned rats were discarded. These rats were excluded because sufficient bilateral damage was not evident. One control rat showed uniltateral damage to $\mathrm{CN}$ and along the injector track; behavioral data from this rat were also discarded. In the six rats with acceptable lesions, the $\mathrm{CN}$ lesions ranged in size from 30 to $70 \%$ of the nucleus, with the average lesion encompassing $\sim 40 \%$ of the nucleus. Medial CN was damaged in all cases (Fig. 1, top right panel). Although these lesions were relatively small, they were found to have significant 

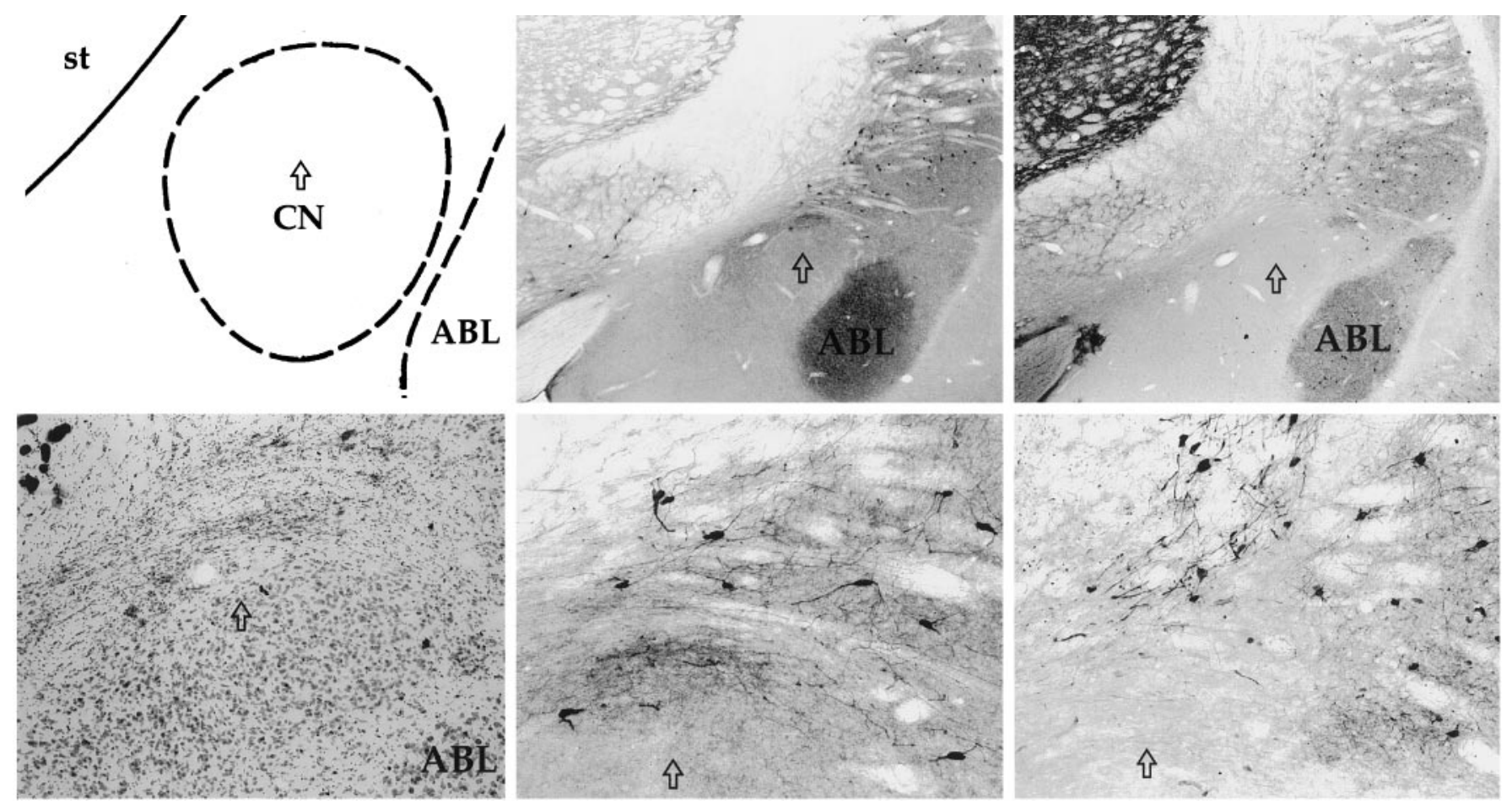

Figure 1. Schematic of the amygdala complex (top left) with the arrow indicating the position of the CN lesion at the level of the sections shown in the photomicrographs. The photomicrograph of a Nissl-stained section (bottom left) reveals heavy gliosis at the lesion site in the dorsal region of CN. Photomicrographs (center panels) show intact ChAT-immunopositive cholinergic neurons surrounding CN at two magnifications. Similarly, photomicrographs (right panels) show intact parvalbumin-immunopositive GABAergic neurons surrounding CN. $A B L$, Basolateral nucleus; st, striatum.

effects on task performance. Cholinergic neurons with ChAT immunostaining and GABAergic neurons with parvalbumin immunostaining were evident throughout $\mathrm{BF}$ in both control and lesioned brains. Importantly, no difference in the pattern and relative density of acetycholinestrase staining was detected in comparing $\mathrm{CN}$ lesioned brains with control brains. In addition, as shown in Figure 1, cholinergic neurons labeled with ChAT antibody and GABAergic neurons labeled with parvalbumin antibody were clearly visible in close proximity to the area of gliosis because of removal of $\mathrm{CN}$ neurons.

\section{Behavioral results}

By both speed and accuracy measures of correct responding, rats with $\mathrm{CN}$ lesions were somewhat slower to learn the three-choice task than control rats $\left(F_{(1,11)} \geq 7.22 ; p<0.02\right)$. In contrast, measures of error responses and anticipatory responses showed no effects of the lesion at any point in training $(F<1)$. There were very few errors of omission; at least one response (correct or error) occurred during target presentation or the subsequent response window on $97.3 \%$ of the training trials in lesioned rats and on $98.1 \%$ of the trials in control rats.

By the last three sessions of training with $0.5 \mathrm{sec}$ target cues, there were no differences between lesioned and control rats in any response measure $(F<1$; Table 1$)$.

\section{Attentional challenges}

Figure 2 shows the effects of the three intended attentional challenges on the latency to the first correct response, and Table 2 shows those effects on the percentage of trials on which the first response was correct. Figure $2 a$ and Table 2, top portion, show performance during the final training session with $0.5 \mathrm{sec}$ targets and the first session with $0.25 \mathrm{sec}$ targets. Reducing the target duration compromised performance in the lesioned but not the control rats. ANOVAs showed a reliable lesion $\times$ duration interaction $\left(F_{(1,11)} \geq 7.58 ; p \leq 0.019\right)$ but no reliable main effect of either lesion $\left(F_{(1,11)} \leq 3.06 ; p \geq 0.108\right)$ or duration $\left(F_{(1,11)} \leq 2.37\right.$; $p \geq 0.152)$. Separate contrasts of the effect of lesion were reliable for the $0.25 \mathrm{sec}$ target cues $\left(F_{(1,11)} \geq 6.59 ; p \leq 0.026\right)$ but not for the
Table 1. Performance in final three training sessions

\begin{tabular}{lcc} 
& Control rats & Lesioned rats \\
\hline Latency to first correct response(s) & $1.84 \pm 0.16$ & $1.82 \pm 0.14$ \\
Trials with a correct response (\%) & $88.3 \pm 1.1$ & $89.8 \pm 1.9$ \\
Trials with correct response first (\%) & $75.6 \pm 2.1$ & $74.7 \pm 1.9$ \\
Latency to first error(s) & $3.83 \pm 0.09$ & $3.78 \pm 0.11$ \\
Trials with an error response (\%) & $88.6 \pm 3.8$ & $87.9 \pm 7.0$ \\
No. of anticipatory responses/trial & $2.4 \pm 0.3$ & $2.5 \pm 0.3$ \\
Latency to first anticipatory response(s) & $4.12 \pm 0.39$ & $4.10 \pm 0.39$
\end{tabular}

Values are mean \pm SEM.

baseline, $0.5 \mathrm{sec}$ target cues $(F<1)$. Reduction in target duration had no reliable effects on errors, anticipatory responding, or the percentage of trials on which at least one correct response occurred (data not shown; $F<1.2$ ).

The deleterious effect of the reduction in target duration on the performance of lesioned rats was short-lived, as indicated by the performance during the second session with $0.25 \mathrm{sec}$ target cues. Figure $2 b$ and Table 2, middle portion, show performance during that session as well as during the first session with $0.25 \mathrm{sec}$ target cues with distracters added in the ready period. Lesion by distracter (no distracter baseline vs distracter session) ANOVAs showed no reliable effect of lesion $\left(F_{(1,11)} \leq 1.61 ; p \geq 0.230\right)$, distracter $(F<1)$, or their interaction $(F<1)$ on any of the latency or accuracy measures of either correct or error responding or on anticipatory responding $(F<1.3)$.

Figure $2 c$ and Table 2, bottom portion, show performance during the first test session with variable $(1,5$, or 9 sec) ready signals as well as during the immediately preceding baseline session with constant 5 sec ready signals. Performance was disrupted by the introduction of variable ready times in both lesioned and control rats, but the disruption was substantially greater in lesioned rats. ANOVAs with lesion condition and test trial type as variables showed reliable main effects of lesion $\left(F_{(1,11)} \geq 6.69 ; p \leq 0.025\right)$ and 

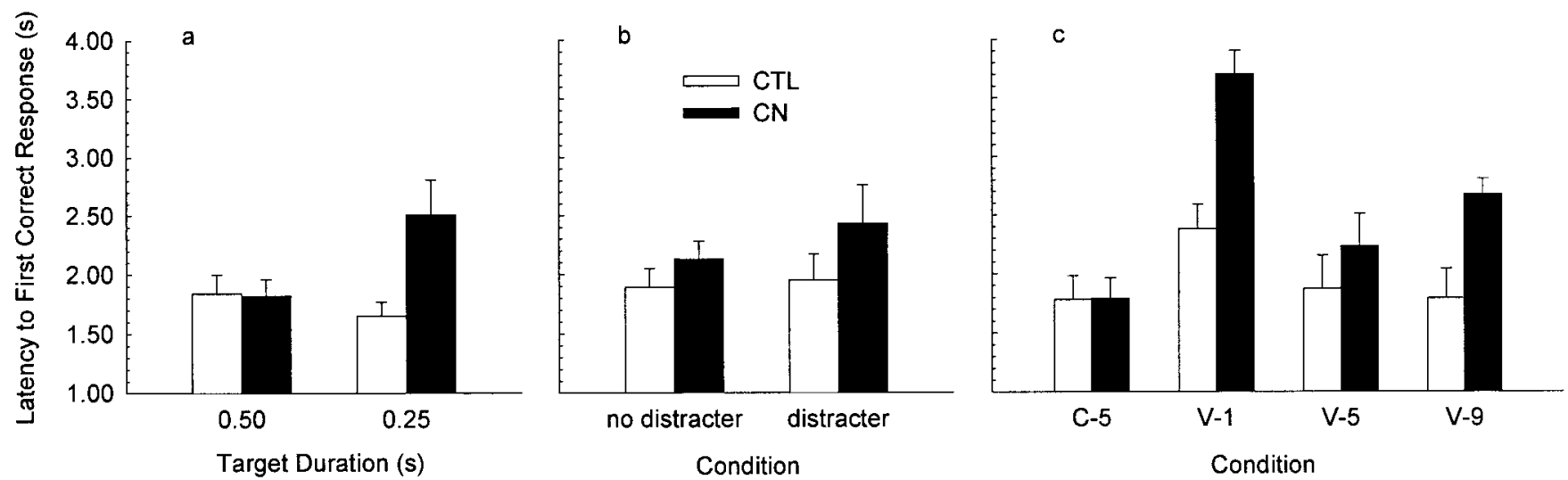

Figure 2. Latencies to the first correct response in the behavioral tests. The filled bars show performance of CN-lesioned rats, and the open bars show performance of control $(C T L)$ rats. $a$, Responding when the target duration was reduced to 0.25 sec and during the immediately preceding baseline session with $0.50 \mathrm{sec}$ targets. $b$, Responding when distracter stimuli were presented during the trials and during the immediately preceding baseline session. $c$, Responding in a test session in which the ready signals were of variable duration $(V-1,1 \mathrm{sec} ; V-5,5 \mathrm{sec}, V-9,9 \mathrm{sec})$ and responding in the immediately preceding baseline session in which the ready signal was always $5 \sec (C-5)$.

\begin{tabular}{|c|c|c|}
\hline Challenge & Control rats & Lesioned rats \\
\hline \multicolumn{3}{|l|}{ Target duration } \\
\hline $0.50 \mathrm{sec}$ & $78.5 \pm 2.2$ & $80.0 \pm 2.4$ \\
\hline $0.25 \mathrm{sec}$ & $77.1 \pm 3.2$ & $67.6 \pm 1.4$ \\
\hline \multicolumn{3}{|l|}{ Distracter } \\
\hline No distracter & $76.1 \pm 4.2$ & $74.0 \pm 4.1$ \\
\hline Distracter & $72.4 \pm 4.6$ & $66.3 \pm 4.8$ \\
\hline \multicolumn{3}{|c|}{ Ready signal variability } \\
\hline Constant, $5 \mathrm{sec}$ & $76.0 \pm 3.8$ & $74.5 \pm 4.2$ \\
\hline Variable, $1 \mathrm{sec}$ & $50.0 \pm 3.5$ & $36.4 \pm 3.2$ \\
\hline Variable, $5 \mathrm{sec}$ & $80.3 \pm 4.7$ & $66.9 \pm 4.2$ \\
\hline Variable, $9 \mathrm{sec}$ & $74.5 \pm 3.6$ & $57.5 \pm 4.1$ \\
\hline
\end{tabular}

Values are mean \pm SEM.

trial type $\left(F_{(3,33)} \geq 14.98 ; p \leq 0.001\right)$ and reliable interactions of those two variables $\left(F_{(3,33)} \geq 4.27 ; p \leq 0.012\right)$. In control rats, performance was poorer on trials with the short $(1 \mathrm{sec})$ ready signal than on any of the other trial types $\left(F_{(1,11)} \geq 5.08 ; p \leq 0.046\right)$ but did not vary across the other trial types $(F<1)$. In lesioned rats, performance during the baseline session with constant $5 \mathrm{sec}$ ready times (C-5 trials) was reliably better than performance on $1 \mathrm{sec}$ ready-time trials in the variable session $(F \geq 44.64 ; p \leq 0.001)$ and 9 sec variable trials $\left(F_{(1,11)} \geq 8.30 ; p \leq 0.015\right)$ and marginally better than on $5 \mathrm{sec}$ ready time trials in the variable session $(0.05<p<$ $0.10)$. Lesioned rats showed poorer performance than control rats on both $1 \sec \left(F_{(1,11)} \geq 17.22 ; p \leq 0.002\right)$ and $9 \sec \left(F_{(1,11)} \geq 7.20\right.$; $p \leq 0.022)$ trials in the variable session but not on 5 sec trials in either the variable or baseline session $(F<1)$. As described later, the percentage of trials on which correct responses occurred was reduced on $1 \mathrm{sec}$ trials in both lesioned and control rats. Consequently, we were concerned that differences in latency to the first correct response may have reflected this failure to respond correctly more than changes in the speed of correct responses actually made. However, analyses of the latency of correct responses confined to trials on which a correct response did occur and those confined to trials on which the correct response was the first response to occur showed patterns of significance essentially identical to those just reported.

Unlike the duration effect shown in Figure $2 a$, the effects of introducing variation in the ready times were persistent. Performance in the second variable test session (data not shown) was essentially identical to that in the first test. Nevertheless, performance recovered immediately in the final test session, in which the rats were returned to the $5 \mathrm{sec}$ constant ready-time baseline. The mean latency to the first correct response in that session was $1.65 \pm$ $0.15 \mathrm{sec}$ in the lesioned rats and $1.63 \pm 0.11 \mathrm{sec}$ in the control rats, and the percentage of trials with the first response a correct one was $80.1 \pm 3.5 \%$ in the lesioned rats and $81.2 \pm 4.0 \%$ in the control rats $(F<1)$.

The introduction of variable ready times also affected several of the other response measures. Not surprisingly, there were more anticipatory responses per trial when the ready times were extended to $9 \mathrm{sec}$ and fewer when they were shortened to $1 \mathrm{sec}\left(F_{(1,11)}\right.$ $\geq 7.37 ; p<0.02$ ). However, unlike with target responding, anticipatory performance of lesioned and control rats was identical $(F<1)$. Control rats emitted an average of $0.3 \pm 0.2$ responses in the $1 \mathrm{sec}$ ready periods and $2.9 \pm 0.2$ responses in the $9 \mathrm{sec}$ ready periods, and lesioned rats emitted $0.2 \pm 0.2$ and $2.8 \pm 0.4$ responses, respectively. This pattern of responding would be expected from the evidence for timing shown in the final training sessions (Table 1). In those sessions, the first anticipatory response on average occurred just before the scheduled time of target presentation in both lesioned and control rats. When the target was delayed in the variable test session, responding began at approximately the same time and continued at approximately the same rate for the next few seconds, as is typically observed in tests of animal timing performed using the "peak procedure" (Roberts, 1981). In addition, both control and lesioned rats emitted marginally more anticipatory responses during the $5 \mathrm{sec}$ ready time trials in the variable session $(2.4 \pm 0.3$ and $1.9 \pm 0.3$, respectively) than in the constant session $\left(1.5 \pm 0.6\right.$ and $\left.1.3 \pm 0.5 ; F_{(1,11)}=3.37 ; p=0.09\right)$.

The percentage of trials with at least one correct response dropped to $61.9 \pm 4.6$ in control rats and $57.8 \pm 9.1$ in lesioned rats on $1 \mathrm{sec}$ ready-time trials compared with $84.5 \pm 4.6$ and $78.6 \pm 6.4$ on the remaining trial types. ANOVA and subsequent contrasts showed no effect of lesion $\left(F_{(1,11)}=1.02\right)$ but significantly lower responding on the $1 \mathrm{sec}$ trials $\left(F_{(1,11)}=21.38 ; p<0.001\right)$. Finally, although the percentage of trials on which an error occurred did not differ across lesion condition or trial type $(F<1)$, the latency to the first error was longer on $1 \mathrm{sec}$ trials than on the remaining trial types in both lesioned rats $(4.24 \pm 0.14$ and $3.65 \pm 0.09 \mathrm{sec}$, respectively) and control rats $(4.33 \pm 0.07$ and $3.69 \pm 0.04 \mathrm{sec}$; $\left.F_{(1,11)}=25.33 ; p<0.001\right)$. Thus, reduction of the ready time slowed all responding to the targets.

\section{Satiation}

Performance of rats in the postsatiation test showed across-theboard reductions in performance compared with performance in the presatiation baseline session $(p<0.01)$. None of these reductions involved lesion effects $(F<1)$. For example, after satiation, control rats failed to respond (with either a correct response or an 
error) on $20.5 \pm 2.3 \%$ of the trials, and lesioned rats failed to respond on $21.7 \pm 2.8 \%$ of the trials, compared with $<1 \%$ before satiation. Likewise, the latency to the first correct response was $3.40 \pm 0.24 \mathrm{sec}$ in the control rats and $3.31 \pm 0.34 \mathrm{sec}$ in the lesioned rats.

\section{DISCUSSION}

Rats with CN lesions performed as well as control rats at the end of training and in a number of return-to-baseline sessions during the study but were especially susceptible to two attentional challenges. The reduction of target duration and the introduction of variability in the duration of the ready signal both reduced the accuracy and speed of target responding. These lesion effects are interpretable as deficits in the rats' ability to direct responding selectively to the appropriate elements in the stimulus array under conditions of increased attentional load superimposed on performance of the well-learned MCRT task. The observations that CN damage did not affect the depressive effects of satiation, the timing of anticipatory responses, or any aspect of performance in the baseline test sessions make it unlikely that the lesion effects were mediated by deficits in motivational or response production mechanisms. Previous studies from our laboratories showed $\mathrm{CN}$ to be critically involved in conditioned orienting (Gallagher et al., 1990) and in the surprise-induced enhancement of CS associability (Holland and Gallagher, 1993a,b; Han et al., 1999). Taken together with those earlier findings, the present results indicate that amygdala $\mathrm{CN}$ may be important in a wide range of attentional processes, including both those involved in the acquisition of new learning and those involved in directing action.

Because our MCRT training procedures were somewhat different from those typically used (see Materials and Methods), and our lesions were made before (rather than after) training on that task, it is difficult to compare our results directly with those of other lesion studies of MCRT performance. Nevertheless, our results can be viewed as analogous to those reported after damage to the $\mathrm{BF}$ system. BF lesions, made after training MCRT performance, produce an initial deficit in baseline performance followed by recovery to control levels after retraining (Robbins et al., 1989; Muir et al., 1994, 1996; Waite et al., 1999). This pattern parallels our observation of $\mathrm{CN}$ lesion deficits in the initial acquisition, but not final performance, of the MCRT task. Likewise, Muir et al. (1994, 1996), Robbins et al. (1989), and Waite et al. (1999) found that rats with damage to $\mathrm{BF}$ were especially sensitive to disruption by variation in the intertrial interval (corresponding to our ready-time interval). Also, as in our study, these investigators found no effect of BF damage on anticipatory responding in most cases. Similarly, Muir et al. (1994, 1996) found that rats with BF damage showed greater deficits than controls when the duration of the target cue was reduced, although Waite et al. (1999) showed no lesion effects with this manipulation. Unlike these other investigators, we did not find reliable lesion effects on performance when distracter stimuli were introduced. However, also unlike Muir et al. (1994, 1996) (but like Waite et al., 1999), our distracter manipulation had no effect on the performance of control rats.

Deficits in MCRT performance after BF lesions have been attributed to removing cholinergic neurons that innervate cortex. The histological examination of the brains of the rats in the current study showed that $\mathrm{BF}$ magnocellular cholinergic neurons were spared in the presence of $\mathrm{CN}$ neuron loss. The BF cholinergic system, however, receives innervation from CN (Groves, 1988), and it seems reasonable to suggest, by analogy, that the role of $\mathrm{CN}$ in MCRT performance is mediated by its connections with SI/ nBM. Using a disconnection lesion procedure, Han et al. (1999) showed that CS associability enhancements depend on the integrity of the connections of $\mathrm{CN}$ with $\mathrm{SI} / \mathrm{nBM}$. It is possible that the role of $\mathrm{CN}$ in both aspects of attentional processing is mediated by these connections. If so, then $\mathrm{CN}-\mathrm{SI} / \mathrm{nBM}$ disconnection lesions would also be expected to disrupt MCRT performance.

$\mathrm{BF}$ cholinergic systems, including $\mathrm{SI} / \mathrm{nBM}$, are frequently assigned a role in the modulation of cortical attentional function
(Voytko, 1996; Everitt and Robbins, 1997; Sarter and Bruno, 1997). Indeed, performance in MCRT tasks is disrupted by lesions of a number of cortical regions as well as by lesions of SI/nBM. For example, Muir et al. (1996) found lesions of the medial frontal cortex (which receives heavy projections from $\mathrm{SI} / \mathrm{nBM}$ ) to have essentially the same effects on performance in the MCRT task as $\mathrm{SI} / \mathrm{nBM}$ lesions. It is tempting to speculate that $\mathrm{CN}$ might ultimately influence cortical attention circuitry involved in sustained and selective attention tasks via its connections with SI/nBM. This possibility is consistent with evidence from studies of associability enhancements. These attentional changes are dependent not only on the connectivity of CN and SI/nBM (Han et al., 1999) but also on projections from $\mathrm{SI} / \mathrm{nBM}$ to posterior parietal cortex (Bucci et al., 1998). In this context, it would be especially interesting to examine the role of medial frontal cortex in associability changes and that of posterior parietal cortex in performance on selective attention tasks. Although Muir et al. (1996) found no effects of lesions of the parietal cortex on MCRT task performance, their lesions explicitly excluded the posterior subregion identified in the research of Bucci et al. (1998, 1999).

Considerable research, for the most part conducted in nonhuman primates, has revealed that the regulation of attention is an integral dynamic property of neural networks in cortex (for review of recent research, see Behrmann and Haimson, 1999). At the same time, increasing evidence points to a role for subcortical systems in the regulation of cortical processing in attention. The examination of such circuitry is likely to be important for understanding the normal operation of attention, as well as disorders in which attention is impaired. For example, certain evidence points to a loss of integrity in the basal forebrain cholinergic system as a basis for deficits in attention in aging and Alzheimer's disease (Parasuraman and Haxby, 1993). Study of the role of the amygdala central nucleus, which may regulate basal forebrain cholinergic neurons in a broad range of attentional functions, may provide an additional basis for understanding these and other disorders, as well as a possible entry into the development of new therapeutic interventions.

\section{REFERENCES}

Behrmann M, Haimson C (1999) The cognitive neuroscience of visual attention. Curr Opin Neurosci 9:158-163.

Bucci DJ, Holland PC, Gallagher M (1998) Removal of cholinergic input to rat posterior parietal cortex disrupts incremental processing of conditioned stimuli. J Neurosci 18:8038-8046.

Bucci DJ, Conley M, Gallagher M (1999) Thalamic and basal forebrain cholinergic connections of the rat posterior parietal cortex. NeuroReport 10:941-945.

Bushnell PJ (1998) Behavioral approaches to the assessment of attention in animals. Psychopharmacology 138:231-259.

Carli M, Robbins TW, Evenden JL, Everitt BJ (1983) Effects of lesions to ascending noradrenergic neurones on performance of a 5-choice serial reaction task in rats: implications for theories of dorsal noradrenergic bundle function based on selective attention and arousal. Behav Brain Res 9:361-380.

Chiba AA, Bucci DJ, Holland PC, Gallagher M (1995) Basal forebrain cholinergic lesions disrupt increments but not decrements in conditioned stimulus processing. J Neurosci 15:7315-7322.

Chiba AA, Bushnell PJ, Oshiro WM, Gallagher M (1999) Selective removal of cholinergic neurons in the basal forebrain alters cued target detection. NeuroReport 10:1-5.

Everitt BJ, Robbins TW (1997) Central cholinergic systems and cognition. Annu Rev Psychol 48:649-684.

Gallagher M, Holland P (1992) Understanding the function of the central nucleus: is simple conditioning enough? In: The amygdala: neurobiological aspects of emotion, memory, and mental dysfunction (Aggleton JP, ed), pp 307-321. New York: Wiley.

Gallagher M, Graham PW, Holland PC (1990) The amygdala central nucleus and appetitive Pavlovian conditioning: lesions impair one class of conditioned performance. J Neurosci 10:1906-1911.

Groves EA (1988) Neural associations of the substantia innominata in the rat: afferent connections. J Comp Neurol 177:315-346.

Hall G, Pearce JM (1979) Latent inhibition of a CS during CS-US pairings. J Exp Psychol Anim Behav Process 5:31-42.

Han J-S, McMahan RW, Holland PC, Gallagher M (1997) The role of an amygdalo-nigrostriatal pathway in associative learning. J Neurosci 17:3913-3919.

Han J-S, Holland PC, Gallagher M (1999) Disconnection of amygdala central nucleus and substantia innominata/nucleus basalis disrupts increments in conditioned stimulus processing. Behav Neurosci 113:143-151. 
Hatfield T, Han J-S, Conley M, Gallagher M, Holland P (1996) Neurotoxic lesions of the basolateral, but not central, amygdala interfere with Pavlovian second-order conditioning and reinforcer-devaluation effects. J Neurosci 16:5256-5265.

Holland PC, Gallagher M (1993a) Effects of amygdala central nucleus lesions on blocking and unblocking. Behav Neurosci 107:235-245.

Holland PC, Gallagher M (1993b) Amygdala central nucleus lesions disrupt increments, but not decrements, in CS processing. Behav Neurosci 107:246-253.

Holland PC, Gallagher M (1999) Amygdala circuitry in attentional and representational processes. Trends Cognit Sci 3:65-73.

Karnovsky MJ, Roots L (1964) A "direct-coloring" thiocholine method for cholinesterases. J Histochem Cytochem 12:219-221.

McGaughy J, Kaiser T, Sarter M (1996) Behavioral vigilance following infusions of $192 \mathrm{IgG}$-saporin into the basal forebrain: selectivity of the behavioral impairment and relation to cortical AChE-positive fiber density. Behav Neurosci 110:247-265.

Muir JL, Dunnett SB, Robbins TW, Everitt BJ (1992) Attentional functions of the forebrain cholinergic systems: effects of intraventricular hemicholinium, physostigmine, basal forebrain lesions and intracortical grafts on a multiple choice serial reaction time task. Exp Brain Res 89:611-622.

Muir JL, Everitt BJ, Robbins TW (1994) AMPA-induced excitotoxic lesions of the basal forebrain: a significant role for the cortical cholergenic system in attentional function. J Neurosci 14:2313-2325.

Muir JL, Everitt BJ, Robbins TW (1996) The cerebral cortex of the rat and visual attention function: dissociable effects of mediofrontal, cingulate, anterior dorsolateral, and parietal cortex lesions on a five-choice serial reaction time task. Cereb Cortex 6:470-481.
Pang K, Williams MJ, Egeth H, Olton DH (1993) Nucleus basalis magnocellularis and attention: effects of muscimol infusions. Behav Neurosci 107:1031-1038.

Parasuraman R, Haxby JV (1993) Attention and brain function in Alzheimer's disease: a review. Neuropsychology 7:242-272.

Pearce JM, Hall G (1980) A model for Pavlovian learning: variations in the effectiveness of conditioned but not of unconditioned stimuli. Psychol Rev 106:532-552.

Robbins TW, Everitt BJ, Marston HM, Wilkinson J, Jones GH, Page KJ (1989) Comparative effects of ibotenic acid and quisqualic acid induced lesions of the substantia innominata on attentional functions int he rat: further implications for the role of the cholinergic neurons of the nucleus basalis in cognitive processes. Behav Brain Res 35:221-240.

Roberts S (1981) Isolation of an internal clock. J Exp Psychol Anim Behav Process 7:242-268.

Sarter M, Bruno JP (1997) Cognitive functions of cortical acetylcholine: toward a unifying hypothesis. Brain Res Rev 23:28-46.

Voytko ML (1996) Cognitive functions of the basal forebrain cholinergic system in monkeys: memory or attention. Behav Brain Res 75:13-25.

Voytko ML, Olton DS, Richardson RT, Gorman LK, Tobin JR, Price DL (1994) Basal forebrain lesions in monkeys disrupt attention but not learning and memory. J Neurosci 14:167-186.

Waite JJ, Wardlow ML, Power AE (1999) Deficit in selective and divided attention associated with cholinergic basal forebrain immunotoxic lesion produced by 192-saporin; motoric/sensory deficit associated with Purkinje cell immunotoxic lesion produced by OX7-saporin. Neurobiol Learn Mem 71:325-352. 